\title{
Evaluation of mitochondrial toxicity of cadmium in clam Ruditapes philippinarum using iTRAQ-based proteomics ${ }^{\star}$
}

\author{
Chenglong Ji a, b, Zhen Lu ${ }^{\text {a, c }}$, Lanlan Xu ${ }^{\text {a, c }}$, Fei Li ${ }^{\text {a }}$, Ming Cong a , Xiujuan Shan ${ }^{\mathrm{b}}$, \\ Huifeng $\mathrm{Wu}^{\text {a, b, * }}$ \\ ${ }^{a}$ CAS Key Laboratory of Coastal Environmental Processes and Ecological Remediation, Yantai Institute of Coastal Zone Research (YIC), Chinese Academy of \\ Sciences (CAS), Shandong Key Laboratory of Coastal Environmental Processes, YICCAS, Yantai, 264003, PR China \\ ${ }^{\mathrm{b}}$ Laboratory for Marine Fisheries Science and Food Production Processes, Qingdao National Laboratory for Marine Science and Technology, Qingdao, \\ 266237, PR China \\ ${ }^{\mathrm{c}}$ University of Chinese Academy of Sciences, Beijing, 100049, PR China
}

\section{A R T I C L E I N F O}

\section{Article history:}

Received 3 April 2019

Received in revised form

30 April 2019

Accepted 10 May 2019

Available online 15 May 2019

\section{Keywords}

Cadmium

iTRAQ

Proteomics

Mitochondrial toxicity

Ruditapes philippinarum

\begin{abstract}
A B S T R A C T
Cadmium is one of the most serious metal pollutants in the Bohai Sea. Previous studies revealed that mitochondrion might be the target organelle of $\mathrm{Cd}$ toxicity. However, there is a lack of a global view on the mitochondrial responses in marine animals to $\mathrm{Cd}$. In this work, the mitochondrial responses were characterized in clams Ruditapes philippinarum treated with two concentrations ( 5 and $50 \mu \mathrm{g} / \mathrm{L}$ ) of Cd for 5 weeks using tetraethylbenzimidazolylcarbocyanine iodide (JC-1) staining, ultrastructural observation and quantitative proteomic analysis. Basically, a significant decrease of mitochondrial membrane potential $(\triangle \Psi m)$ was observed in clams treated with the high concentration $(50 \mu \mathrm{g} / \mathrm{L})$ of $\mathrm{Cd}$. Cd treatments also induced specific morphological changes indicated by elongated mitochondria. Furthermore, iTRAQbased mitochondrial proteomics showed that a total of 97 proteins were significantly altered in response to $\mathrm{Cd}$ treatment. These proteins were closely associated with multiple biological processes in mitochondria, including tricarboxylic acid (TCA) cycle, oxidative phosphorylation, fatty acid $\beta$-oxidation, stress resistance and apoptosis, and mitochondrial fission. These findings confirmed that mitochondrion was one of the key targets of Cd toxicity. Moreover, dynamical regulations, such as reconstruction of energy homeostasis, induction of stress resistance and apoptosis, and morphological alterations, in mitochondria might play essential roles in Cd tolerance. Overall, this work provided a deep insight into the mitochondrial toxicity of $\mathrm{Cd}$ in clams based on a global mitochondrial proteomic analysis.
\end{abstract}

(c) 2019 Elsevier Ltd. All rights reserved.

\section{Introduction}

With the rapid development of industry, cadmium (Cd) has become one of the most serious metal pollutants in the Bohai Sea because of a huge amount of Cd discharge from industrial effluents (Ji et al., 2016; Luo et al., 2013). Many surveys, including those by our group, have demonstrated Cd pollution in multiple environmental media in the Bohai Sea (Gao et al., 2014; Ji et al., 2016; Xu et al., 2016a). Based on the published data, the seawater $\mathrm{Cd}$

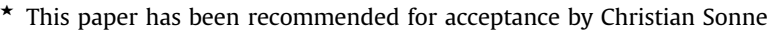

* Corresponding author. CAS Key Laboratory of Coastal Environmental Processes and Ecological Remediation, Yantai Institute of Coastal Zone Research (YIC), Chinese Academy of Sciences (CAS); Shandong Key Laboratory of Coastal Environmental Processes, YICCAS, Yantai, 264003, PR China.

E-mail address: hfwu@yic.ac.cn (H. Wu).
}

concentration has reached to $5 \mu \mathrm{g} / \mathrm{L}$ in the Jinzhou Bay and $16 \mu \mathrm{g} / \mathrm{L}$ in the Liaodong Bay in the Bohai Sea, respectively (Ma et al., 1995, Zhang et al., 2001). Moreover, our findings showed that Cd was one of the major metal pollutants accumulated in shrimp Crangon affinis that is a representative benthic species in the Bohai Sea (Xu et al., 2016a). These evidences confirm the historic contamination of $\mathrm{Cd}$ in the Bohai Sea, and the threats of $\mathrm{Cd}$ to marine organisms certainly deserve to be further concerned.

Increasing studies have been conducted on the threats of $\mathrm{Cd}$ to marine organisms, demonstrating that Cd could inhibit growth, disrupt energy metabolism, and induce immune and oxidative stress in marine organisms (Fernandez-Leborans and Herrero, 1999; Ji et al., 2015a; Qu et al., 2013; Sokolova, 2004; Wang and Rainbow, 2006; Wu et al., 2017). Especially, our previous work showed that Cd could affect energy metabolism in marine bivalves, indicated by the alterations of metabolites including glucose, amino 
acids, ATP, ADP, and other intermediates in tricarboxylic acid (TCA) cycle (Ji et al., 2015a; Wu et al., 2017). The mechanisms of Cd toxicity have been widely but fully explored. Based on so-called ionic mimicry, $\mathrm{Cd}$ exerts its toxic effects by replacing elements like calcium $\left(\mathrm{Ca}^{2+}\right)$ and trace elements like zinc $\left(\mathrm{Zn}^{2+}\right)$ (Choong et al., 2014). The overproduction of reactive oxygen species (ROS) was also reported to be induced by $\mathrm{Cd}$ via binding to the thiol groups of proteins, subsequently leading to oxidative stress, DNA damage, lipid peroxidation, and even apoptosis (Chen et al., 2015; Gobe and Crane, 2010). Interestingly, all these evidences suggested that mitochondrion should be the target organelle of Cd toxicity.

As it is known, mitochondria are dynamic organelles responsible for ATP production through oxidative phosphorylation (OXPHOS) and play an essential role in a diverse array of metabolic processes, such as apoptosis, calcium h cycle, fatty acid metabolism, amino acid metabolism, and heme biosynthesis (Meyer et al., 2013). Considering the complexity of mitochondria, it is not surprising that they are often deemed as the target of pollutant-induced toxicity (Meyer et al., 2013). The mitochondrial toxicity of Cd has been reflected in influencing mitochondrial bioenergetics and gene expressions of key mitochondrial proteins, as well as causing oxidative stress (Gobe and Crane, 2010; Sokolova et al., 2005). For example, Sokolova et al. (2005) observed that Cd can be accumulated in mitochondria of oyster, which subsequently led to impaired capacity for ATP production. Though the toxicological effects of Cd to mitochondria have been confirmed, there is an obvious lack of global characterization of mitochondrial responses to $\mathrm{Cd}$. Hence, a comprehensive evaluation of mitochondrial responses is vital to further understanding the mitochondrial toxicity of Cd.

Intertidal organisms, including mollusks, crustaceans and fishes, are vulnerable to environmental insults and mainly rely on mitochondria to adapt exogenous stresses (Sokolova, 2018). Clam Ruditapes philippinarum is a typical intertidal species in the Bohai Sea, which has been frequently used as a preferable indicator in pollution biomonitoring because of its wide distribution and high tolerance to pollutants (Ji et al., 2015b; Liu et al., 2011; Marques et al., 2018). Proteomics that focuses on the global changes of proteome has been frequently used to characterize the toxicological mechanisms of pollutants. Our previous work using proteomics approach revealed that $\mathrm{Cd}$ could disturb energy metabolism and induce oxidative stress in bivalves, implying that mitochondrion might be the target of Cd (Xu et al., 2016b). Considering the importance of mitochondria and its susceptibilities to $\mathrm{Cd}$, it is necessary to characterize the global response of mitochondria to $\mathrm{Cd}$ at protein level. Mitochondrial proteomics is capable of providing new insights into mitochondrial function, and has been increasingly used in studies on human disease and ageing (Calvo and Mootha, 2010). To our knowledge, few studies have focused on the global characterization, at protein level, of mitochondrial responses of clams to $\mathrm{Cd}$ treatment. In the present study, highthroughput proteome screening based on iTRAQ technique was conducted to detect the responsive proteins in clam mitochondria, aiming to unravel the strategy of mitochondrial responses of clams to $\mathrm{Cd}$ treatment.

\section{Materials and methods}

\subsection{Animals and experimental design}

Adult clams $R$. philippinarum (shell length: $3.5-3.8 \mathrm{~cm}$, White pedigree) were purchased from a culturing farm (Qingdao, China) and acclimated in aerated seawater in laboratory for 7 days. After acclimation, animals were then randomly divided into three groups (control group and two Cd-treated groups). The clams cultured in the normal filtered seawater were used as control group. Other two groups of clams were exposed to two sublethal concentrations ( 5 and $50 \mu \mathrm{g} / \mathrm{L}$ ) of $\mathrm{Cd}$ by the addition of stock solutions of $\mathrm{CdCl}_{2}$, which were prepared using deionized water and analytical reagent grade compounds. Each treatment consisted of 30 individuals and was performed in triplicate per group. Seawater was completely replaced by fresh seawater every day. During the acclimation and exposure periods, clams were kept in aerated seawater $\left(25 \pm 2{ }^{\circ} \mathrm{C}\right.$, $31 \mathrm{ps} \mu$ ) under a photoperiod of $12 \mathrm{~h}$ light and $12 \mathrm{~h}$ dark, fed with Chlorella vulgari of $2 \%$ tissue dry weight once daily. After exposure for 35 days, gill tissues of clams were immediately dissected for subsequent analyses.

\subsection{Isolation of mitochondria}

Mitochondria were isolated from gills according to (Li et al., 2009) with some modifications. To keep the mitochondria alive, all steps were performed at $4{ }^{\circ} \mathrm{C}$. In brief, the fresh gill tissues were transferred to pre-cooled tubes, followed by Dounce homogenization (20 strokes) in lysis buffer I (LB I: $250 \mathrm{mM}$ sucrose, $10 \mathrm{mM}$ HEPES, 1 mM EGTA, pH 7.4) containing protease inhibitor cocktail (Roche Applied Science, Mannheim, Germany). The homogenate was centrifuged at $1500 \mathrm{~g}$ for $10 \mathrm{~min}$, and the supernatant was reserved. The pellet was re-suspended in lysis buffer and the centrifugation at $1500 \mathrm{~g}$ for $10 \mathrm{~min}$ was repeated twice more. The supernatants were combined and treated with an additional centrifugation at $10,000 \mathrm{~g}$ for $10 \mathrm{~min}$. The pellet containing crude mitochondria were re-suspended and stored in LB I. Further enrichment was conducted on the crude mitochondria by centrifugation at a serial of Nycodenz ${ }^{\mathbb{R}}$ gradients at $40,000 \mathrm{~g}$ for $45 \mathrm{~min}$. The gradient solutions $(0.5 \mathrm{~mL}$ of $20 \%, 2 \mathrm{~mL}$ of $23 \%, 2.5 \mathrm{~mL}$ of crude mitochondria in $25 \%, 2 \mathrm{~mL}$ of $30 \%$ and $1.2 \mathrm{~mL}$ of 34 and $40 \%$ ) were prepared from $80 \%$ Nycodenz $^{\circledR}$ stock (10 mM HEPES, 1 mM EGTA, $\mathrm{pH}$ 7.4). The pure mitochondria fraction with a pale yellow color between 25 and 30\% gradients was carefully collected and washed with LB I, and then stored in lysis buffer II (9 M urea, 4\% CHAPS, 1\% $\mathrm{w} / \mathrm{v}$ DTT) for further proteomic analysis.

\subsection{Mitochondrial morphology by transmission electron microscopy}

Transmission electron microscopy (TEM) of gill mitochondria was carried out to evaluate the morphological alterations of mitochondria in gills between control and Cd-treated clams. Detailed descriptions on production and examination of sections were available in the Supporting Information S1.

\subsection{Measurement of mitochondrial membrane potential}

The assessment of mitochondrial membrane potential (MMP, $\triangle \Psi m$ ) was conducted using a commercial kit (Beyotime, Nantong, China) based on the mitochondrial-specific fluorescent probe tetraethylbenzimidazolylcarbocyanine iodide (JC-1). In mitochondria with high $\triangle \Psi m$, JC-1 spontaneously forms complexes known as Jaggregates with intense red fluorescence (590 nm). Oppositely, JC-1 remains in monomeric form in mitochondria with low $\triangle \Psi m$, presenting green fluorescence $(527 \mathrm{~nm})$. In brief, each crude mitochondria sample isolated from gills of 9 clams was mixed into JC-1 reagent with the volume ratio of 9 to 1 . The mixtures were detected immediately using BD FACSAria flow cytometer (Becton, $\mathrm{NJ}$, USA) at $490 \mathrm{~nm}$ excitation. JC-1 monomers and aggregates showing green and red spectral shifts were measured in FL1 channel (515-545 nm) and FL2 channel (562-588 nm), respectively. Data were then processed using FACSAria Diva software 6.1.3 and plotted on histogram of FL2 versus FL1, and the ratio of FL2/FL1 for JC-1 is generally used to indicate $\triangle \Psi m$ in stained mitochondria. 
The ratio was shown as mean \pm standard deviation $(n=4)$. One way analysis of variance (ANOVA) was conducted to evaluate the difference of ratios between control and Cd treatment groups, and a $p$ value less than 0.05 was considered statistically significant.

\subsection{Proteomic analysis}

Each pure mitochondria sample isolated from gills of 13 clams was dissolved in lysis buffer ( $9 \mathrm{M}$ urea, 4\% CHAPS, $1 \% \mathrm{w} / \mathrm{v}$ DTT) with protease inhibitor (Roche Applied Science, Mannheim, Germany). The concentrations of the protein extracts were determined using the Bradford method (Bradford, 1976). iTRAQ technique was applied for quantitative proteomic analysis. Briefly, $100 \mu \mathrm{g}$ of protein extracted from each sample was dissolved in the dissolution buffer (AB SCIEX, MA, USA). After being reduced, alkylated and trypsin-digested, the protein samples were labeled using iTRAQ 4plex reagents (AB SCIEX, MA, USA) according to the manufacturer's protocol. Samples from control, low concentration $(5 \mu \mathrm{g} / \mathrm{L})$ of $\mathrm{Cd}$ treatment, and high concentration $(50 \mu \mathrm{g} / \mathrm{L})$ of Cd treatment groups were labeled with 119-, 117-, and 118-tag, respectively. A pooled sample from three groups was labeled with 121-tag for internal normalization. Three biological replicates were used in each group. The peptides were then purified from excess labeling reagent by strong cation exchange (SCX) chromatography using Agilent 1200 HPLC (Agilent, CA, USA), followed by being separated using Eksigent nanoLC-Ultra 2D system (Eksigent, CA, USA). The LC fractions were analyzed by MS/MS using a Triple TOF 5600 mass spectrometer (AB SCIEX, MA, USA).

Protein identification and relative quantification were performed with ProteinPilot Software 5.0 (AB SCIEX). MS/MS data were searched against a mollusk protein database (translated from $R$. philippinarum transcriptome database with 38,224 sequences, built in 2013) and a decoy database for false discovery rate (FDR) analysis. The database search parameters were the followings: iTRAQ 4-plex quantification, cysteine modified with iodoacetamide, methionine modifications of oxidation, trypsin digestion, one missed cleavage allowed, peptide tolerance was set as $0.05 \mathrm{Da}$, and MS/MS tolerance was set as 0.05 Da. Only peptides identified with local FDR $<0.05$ were considered for further analysis. Proteins with unused score $>1.3$, matching reliable peptides ( $\geq 2$ unique peptides) were considered as positively identified proteins. Protein ratio was calculated by averaging the ratios of three replicates, and protein with a fold change ratio $>1.2$ or $<0.83$ as well as a $p$ value $<0.05$ in three replicates was considered as differentially expressed proteins (DEPs). Evaluation of function of DEPs was performed according to NCBI (https://www.ncbi.nlm.nih.gov/), Uniprot (https://www. uniprot.org/) and KEGG (https://www.genome.jp/kegg/) database analyses. More detailed information of iTRAQ analysis, protein identification and quantification was described in the Supporting Information S1.

\subsection{Nuclear magnetic resonance-based metabolomic analysis}

Nuclear magnetic resonance (NMR)-based metabolomics was used to reveal the alterations of metabolites in gill samples of clams treated with Cd. Detailed information was available in Supporting Information S1.

\section{Results and discussion}

\subsection{Mitochondrial membrane potentials $(\triangle \Psi \mathrm{m})$}

Mitochondrial membrane potential $(\triangle \Psi m)$ is primarily generated by proton pumps (Complex I, III, IV) and essential for ATP production (Zorova et al., 2018). It also provides a driving force for transport of ions (except for $\mathrm{H}^{+}$) and proteins which are necessary for mitochondrial function (Zorova et al., 2018). The measurement of $\triangle \Psi m$ based on $\mathrm{JC}-1$ probe has been reported as a suitable candidate for assessing pollution toxicity in fish (Padmini and Rani, 2011). As shown in Fig. 1A, partitions of P2 (red) and P3 (green) represented mitochondria with high and low $\triangle \Psi m$, respectively. The ratio of $\mathrm{P} 2 / \mathrm{P} 3$ was used to indicate $\triangle \Psi m$. The results demonstrated that both concentrations of $\mathrm{Cd}$ caused decreases of $\triangle \Psi m$. However, the significant $(p<0.05)$ alteration of $\triangle \Psi m$ was uniquely found in the high concentration $(50 \mu \mathrm{g} / \mathrm{L})$ of $\mathrm{Cd}$-treated clams. Cd was prone to attach to the inner membrane of mitochondria and enhance the membrane permeability, resulting in $\mathrm{H}^{+}$ efflux (Muller, 1986; Rasheed et al., 1984). In fact, the negative effect of $\mathrm{Cd}$ to $\triangle \Psi \mathrm{m}$ has been frequently reported in cells or isolated mitochondria (Belyaeva et al., 2008; Li et al., 2003). Oyster mitochondria treated with Cd presented a significant decrease of ATP synthesis, which was partially attributed to mitochondrial membrane potential dissipation (Sokolova et al., 2005). Consistently, the decreases of $\triangle \Psi m$ were also observed in clams treated with $\mathrm{Cd}$ in our case, especially in the high concentration $(50 \mu \mathrm{g} / \mathrm{L})$ of $\mathrm{Cd}$ exposed individuals, indicating that $\mathrm{Cd}$ possibly influenced ATP synthesis and healthy function of mitochondria.

\subsection{Global mitochondrial proteome alterations in response to $\mathrm{Cd}$ treatment}

Analyses of three biological replicates resulted in 864, 976 and 930 proteins, respectively (Supporting Information S2). A total of 383 proteins were identified in all three replicates, among which 97 proteins were differentially expressed in response to Cd treatments. Considering that many DEPs were hypothetical proteins with unknown functions, further blast was conducted on DEPs by searching database of Non-redundant protein sequences database (nr) using blastp, resulting in a total of 26 out of 97 DEPs identified as known mitochondrial proteins. In order to characterize the mitochondrial responses of clams to $\mathrm{Cd}$ treatment, we only focused on function analysis of mitochondrial DEPs, which were listed in Table 1. Detailedly, among these 26 DEPs, 21 and 16 DEPs were detected in clams treated with $5 \mu \mathrm{g} / \mathrm{L}$ and $50 \mu \mathrm{g} / \mathrm{L} \mathrm{Cd}$, respectively. In the low concentration $(5 \mu \mathrm{g} / \mathrm{L})$ of $\mathrm{Cd}$-exposed group, there were 6 upregulated and 15 down-regulated DEPs. Three up-regulated and thirteen down-regulated DEPs were identified in clams treated with the high concentration $(50 \mu \mathrm{g} / \mathrm{L})$ of $\mathrm{Cd}$. It is noteworthy that only 9 out of 26 DEPs exhibited common alteration tendency in both Cd-treated groups. The differential proteomic responses implied that there might be dose-dependent effects of $\mathrm{Cd}$ on clam mitochondria.

\subsection{Mitochondrial morphology}

Mitochondrial morphologies have been reported to be often associated with energetic states and survival of cells (Li et al., 2017). Normally, active mitochondria continually alter its shapes, such as fission and fusion, to maintain mitochondrial functions when cells experience metabolic or environmental stresses (Youle and van der Bliek, 2012). In this work, TEM was conducted to characterize the influences of $\mathrm{Cd}$ on the ultrastructure of mitochondria. Representative mitochondrial elongations were observed in clams from both Cd-treated groups (Fig. 2). Interestingly, proteomic analysis demonstrated that several proteins related to regulating mitochondrial morphology were also significantly altered in Cdexposed clams. Dynamin-1 like protein (DLP1) shares up to $43 \%$ homology with dynamin-related protein 1 (DRP1) which plays a critical role in the mitochondrial fission (Kanamaru et al., 2012; Yoon et al., 1998). Evidences have confirmed that down-regulation 


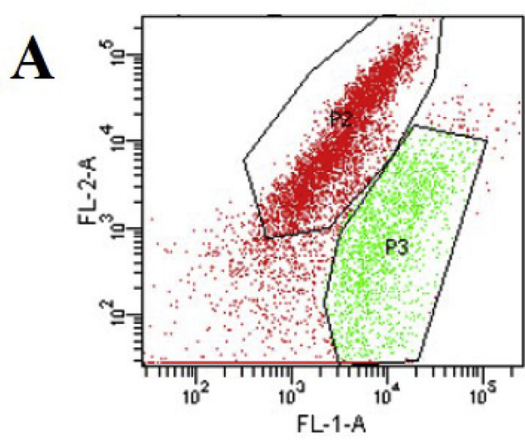

Control

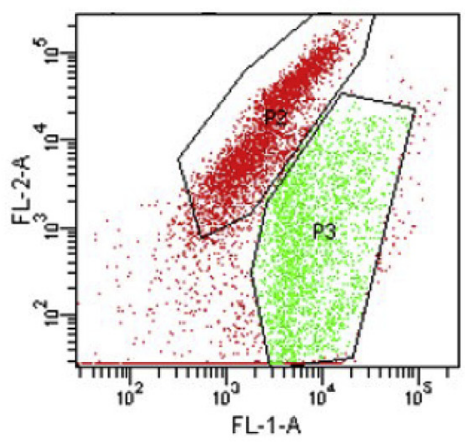

$5 \mu \mathrm{g} / \mathrm{L} \mathrm{Cd}$

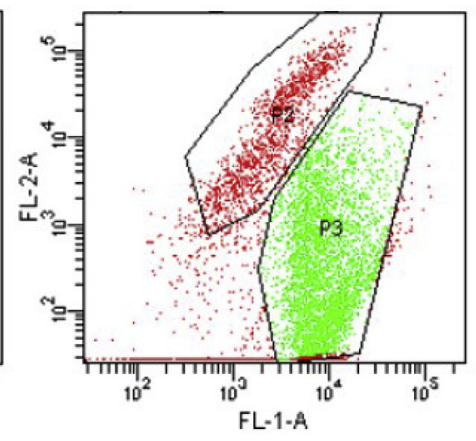

$50 \mu \mathrm{g} / \mathrm{L} \mathrm{Cd}$

B

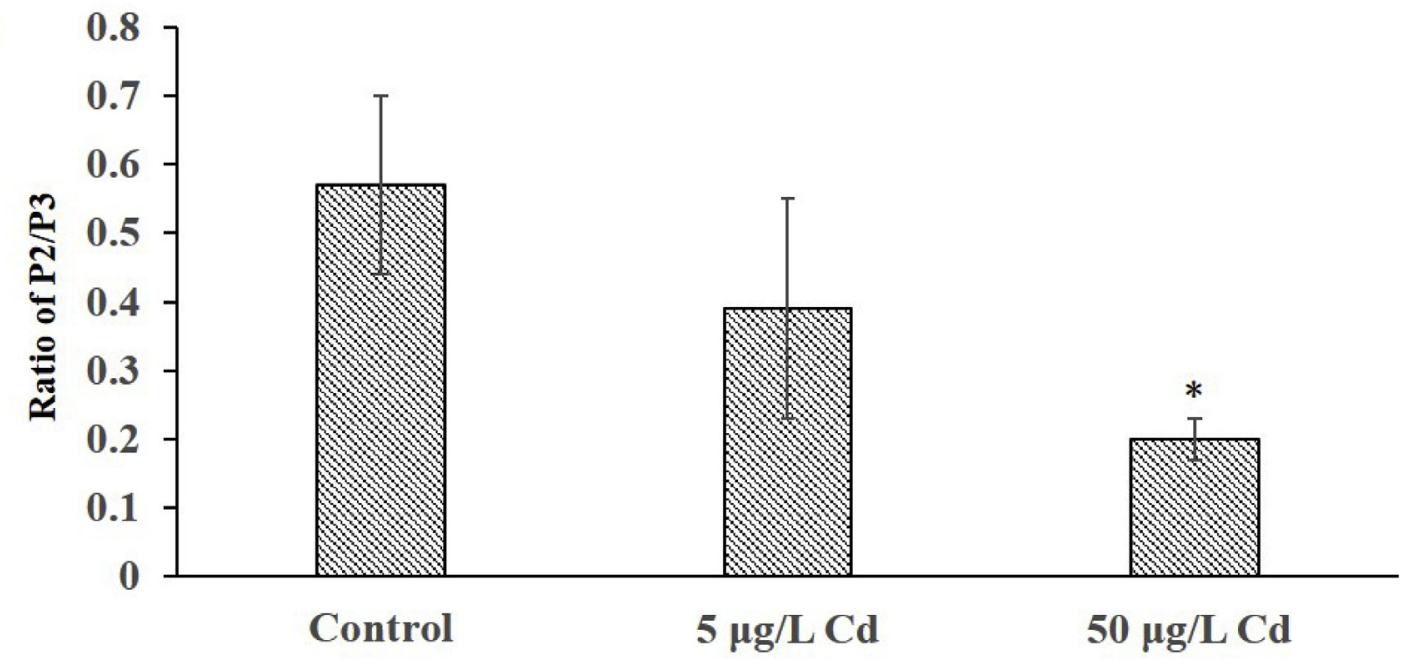

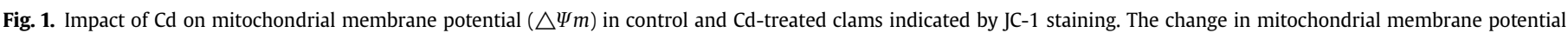

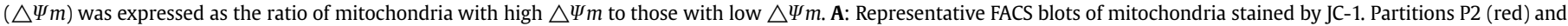

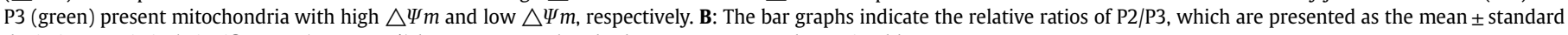
deviation. Statistical significances $\left(p<0.05,{ }^{*}\right)$ between control and $\mathrm{Cd}$ treatments were determined by one way ANOVA.

or inhibition of DRP1 contributed to mitochondrial elongation and swelling by regulating calcium homeostasis (Frank et al., 2001; Mai et al., 2010; Xu et al., 2013; Yu et al., 2016). In this work, the downregulation of DRP1 were highly coincident with mitochondrial elongation in Cd-treated clams. Apart from catalyzing the synthesis of glutamate from proline, delta-1-pyrroline-5-carboxylate dehydrogenase $(\mathrm{P} 5 \mathrm{CDH})$ is also required for mitochondrial integrity. The loss of P5CDH was associated with mitochondrial swelling (He and DiMario, 2011). Moreover, CDGSH iron-sulfur domain-containing protein 2 (CISD2) is well known as a regulator of autophagy, and its abnormal regulation was always linked to environmental stimulus like ocean acidification (Timmins-Schiffman et al., 2014). In this work, P5CDH was significantly down-regulated in clams from both Cd treatments, while decreases of CISD2 and DLP1 were only observed in $5 \mu \mathrm{g} / \mathrm{L}$ and $50 \mu \mathrm{g} / \mathrm{L} \mathrm{Cd-treated} \mathrm{clams,} \mathrm{respectively.} \mathrm{The}$ alterations at protein and ultrastructure levels suggested that mitochondrial morphology was altered to adapt Cd exposure.

\subsection{Energy metabolism}

\subsubsection{TCA cycle}

TCA cycle, also named as citric acid cycle or Krebs cycle, is a central hub of carbon metabolism coordinating the metabolism of glucose, amino acids, respiration, and biosynthetic pathways. It generates co-enzymes like $\mathrm{NADH}$ and $\mathrm{FADH}_{2}$ used in electron transport chain (ETC). In this work, five proteins related to TCA cycle, including $\mathrm{NAD}(\mathrm{P})$ transhydrogenase (nicotinamide nucleotide transhydrogenase, NNT), mitochondrial glutamate dehydrogenase 1 (GLUD1), phosphoenolpyruvate carboxykinase (PEPCK), propionyl-CoA carboxylase alpha (PCCA), and mitochondrial dihydrolipoamide dehydrogenase (DLD), were inhibited by Cd treatments. Among these proteins, NNT, GLUD1 and PEPCK were downregulated in both $\mathrm{Cd}$ treatments, while other two proteins, PCCA and DLD, were decreased in the high concentration $(50 \mu \mathrm{g} / \mathrm{L})$ of $\mathrm{Cd}-$ treated group. NNT catalyzes the transfer of hydride ion equivalents from NADH to NADPH using the proton gradient, whose knockdown inhibited the contribution of glutamine to the TCA cycle and activates glucose catabolism (Gameiro et al., 2013). In this work, Cd treatments caused significant $(p<0.05)$ down-regulations of NNT in clams, which might subsequently weaken glutamine utilization and enhance glucose utilization in TCA cycle. GLUD1 catalyzes the reversible conversion of glutamate to $\alpha$-ketoglutarate. The downregulated GLUD1 caused by Cd treatments implied the alleviation of $\alpha$-ketoglutarate supply to TCA cycle. PEPCK functions as a hub molecule that links TCA cycle intermediates and glycolytic pools through the conversion of mitochondrial oxaloacetate into phosphoenolpyruvate (Stark et al., 2014). The expression of PEPCK has been reported to be negatively regulated by glucose (Vincent et al., 2015). Interestingly, all these proteins, including NNT, GLUD1, and PEPCK, are associated with regulation of glucose and (or) glutamine. Normally, both glucose and glutamine can be involved in TCA cycle by providing intermediates such as acetyl-CoA and $\alpha$ - 
Table 1

The list of differentially expressed proteins in gill mitochondria from clams treated with Cd.

\begin{tabular}{|c|c|c|c|c|c|c|}
\hline Accession & E value & Uniprot & Gene name & Description & $\mathrm{FC} 5^{\mathrm{a}}$ & FC $50^{\mathrm{b}}$ \\
\hline \multicolumn{7}{|l|}{ TCA cycle } \\
\hline c130046_g1 & 0 & P00367 & GLUD1 & Glutamate dehydrogenase 1 , mitochondrial & 0.64 & 0.77 \\
\hline c126333_g1 & 0 & Q16822 & PCK2 & Phosphoenolpyruvate carboxykinase [GTP], mitochondrial & 0.38 & 0.50 \\
\hline c120997_g1 & 0 & Q13423 & NNT & $\mathrm{NAD}(\mathrm{P})$ transhydrogenase, mitochondrial & 0.61 & 0.49 \\
\hline c131875_g1 & 0 & P05165 & PCCA & Propionyl-CoA carboxylase alpha chain, mitochondrial & & 0.51 \\
\hline c124115_g1 & 0 & P09622 & DLD & Dihydrolipoyl dehydrogenase, mitochondrial & & 0.44 \\
\hline \multicolumn{7}{|c|}{ Oxidative phosphorylation } \\
\hline c112081_g1 & $2.00 \mathrm{E}-15$ & Q9Y6M9 & NDUFB9 & $\mathrm{NADH}$ dehydrogenase [ubiquinone] 1 beta subcomplex subunit 9 & 0.69 & \\
\hline c122165_g1 & 8.00E-103 & 075489 & NDUFS3 & NADH dehydrogenase [ubiquinone] iron-sulfur protein 3 & 1.68 & 1.32 \\
\hline c115143_g1 & $1.00 \mathrm{E}-90$ & P47985 & UQCRFS1 & Cytochrome $b-c 1$ complex subunit 11 & 0.53 & \\
\hline c125555_g1 & $5.00 \mathrm{E}-19$ & P22695 & UQCRC2 & Cytochrome $b-c 1$ complex subunit 2 , mitochondrial & 0.58 & \\
\hline c114452_g1 & $3.00 \mathrm{E}-21$ & P20674 & COX5A & Cytochrome $c$ oxidase subunit $5 \mathrm{~A}$, mitochondrial & 2.33 & 0.73 \\
\hline c115262_g1 & $1.00 \mathrm{E}-35$ & P24539 & ATP5F1 & ATP synthase $\mathrm{F}(0)$ complex subunit $\mathrm{B} 1$, mitochondrial & 1.41 & 0.75 \\
\hline c115113_g1 & $1.00 \mathrm{E}-31$ & Р30049 & ATP5D & ATP synthase subunit delta, mitochondrial & & 0.36 \\
\hline c119322_g1 & $4.00 \mathrm{E}-89$ & Q9HAN9 & NMNAT1 & Nicotinamide/nicotinic acid mononucleotide adenylyltransferase 1 & 0.61 & 0.47 \\
\hline c78903_g1 & $4.00 \mathrm{E}-45$ & Q9UIJ7 & AK3 & GTP:AMP phosphotransferase AK3, mitochondrial & 0.42 & \\
\hline c133792_g1 & 0 & P43304 & GPD2 & Glycerol-3-phosphate dehydrogenase, mitochondrial & 0.77 & \\
\hline \multicolumn{7}{|l|}{ Morphology } \\
\hline c131435_g1 & 0 & P30038 & ALDH4A1 & Delta-1-pyrroline-5-carboxylate dehydrogenase & 0.41 & 0.47 \\
\hline c122087_g1 & $7.00 \mathrm{E}-22$ & Q8N5K1 & CISD2 & CDGSH iron-sulfur domain-containing protein 2 & 0.35 & \\
\hline c135035_g1 & $1.00 \mathrm{E}-25$ & 000429 & DNM1L & Dynamin-1-like protein & & 0.74 \\
\hline \multicolumn{7}{|c|}{ Fatty acid $\beta$-oxidation } \\
\hline c130064_g1 & 3.00E-176 & P42765 & ACAA2 & 3-ketoacyl-CoA thiolase, mitochondrial & & 0.70 \\
\hline c134067_g1 & 0 & P40939 & HADHA & Trifunctional enzyme subunit alpha, mitochondrial & 0.61 & 0.54 \\
\hline \multicolumn{7}{|c|}{ Stress resistance and apoptosis } \\
\hline c133979_g1 & $9.00 \mathrm{E}-154$ & Q9NRK6 & ABCB10 & ATP-binding cassette sub-family B member 10 , mitochondrial & 1.32 & 1.20 \\
\hline c129163_g1 & 0 & P05091 & ALDH2 & Aldehyde dehydrogenase, mitochondrial & 1.45 & \\
\hline c131764_g1 & $9.00 \mathrm{E}-146$ & Q9Y6N5 & SQRDL & Sulfide:quinone oxidoreductase, mitochondrial & 0.57 & \\
\hline c133597_g1 & 0 & 000571 & DDX3X & ATP-dependent RNA helicase DDX3X & 0.43 & \\
\hline c124602_g1 & $3.00 \mathrm{E}-138$ & Q9UJZ1 & STOML2 & Stomatin-like protein 2 , mitochondrial & 1.54 & 1.45 \\
\hline c112823_g1 & $4.00 \mathrm{E}-138$ & P45880 & VDAC2 & Voltage-dependent anion-selective channel protein 2 & 0.48 & \\
\hline
\end{tabular}

${ }^{a}, b$ the fold changes of DEPs in the low $(5 \mu \mathrm{g} / \mathrm{L})$ and high $(50 \mu \mathrm{g} / \mathrm{L})$ concentrations of Cd-treated groups, respectively, compared to those in control $(p<0.05)$.
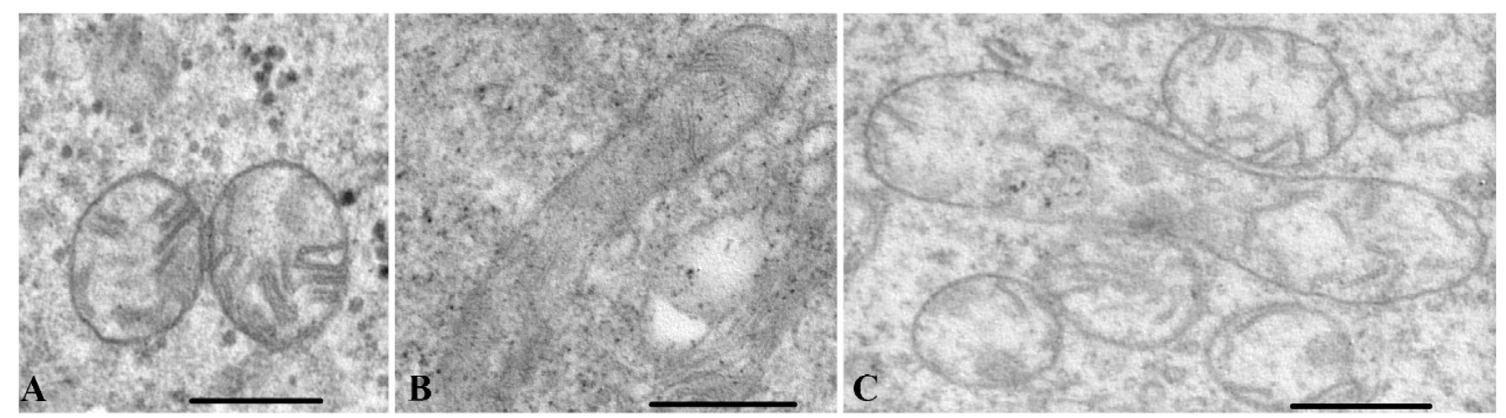

Fig. 2. TEM micrographs of mitochondria from clam gills. A: Control group. B: $5 \mu \mathrm{g} / \mathrm{L}$ Cd treatment group. C: $50 \mu \mathrm{g} / \mathrm{L}$ Cd treatment group. Scale bar: 500 nm.

ketoglutarate. In some cases, glucose and glutamine compensate for each other to maintain TCA cycle function (Yang et al., 2014). In this work, the down-regulations of NNT, GLUD1 and PEPCK were consistently observed in clams treated with $\mathrm{Cd}$, indicating that the pathway linked to TCA cycle via glutamine was alleviated while the pathway via glucose was enhanced. Interestingly, the alterations of these proteins were highly consistent with the increased glucose in Cd-treated clams characterized by metabolomic analysis (Fig. 3, Supporting Information S1, Fig. 1S), confirming the increased demand of glucose for TCA cycle. It is noteworthy that two more proteins, PCCA and DLD, were down-regulated in clams from $50 \mu \mathrm{g} /$ L Cd-treated group. PCAA is linked to producing methylmalonylCoA which is then converted into succinyl-CoA, an intermediate in the TCA cycle (Wongkittichote et al., 2017). DLD is regarded as a critical component of multiple mitochondrial dehydrogenase complexes including pyruvate dehydrogenase complex, $\alpha$-ketoglutarate dehydrogenase complex, and branched chain amino acid (BCAA) dehydrogenase complex. It provides succinyl-CoA and
acetyl-CoA to TCA cycle. The down-regulation of PCCA and DLD signified that the high concentration $(50 \mu \mathrm{g} / \mathrm{L})$ of $\mathrm{Cd}$ might impede the influxes of succinyl-CoA and acetyl-CoA into TCA cycle. That is to say, the high concentration of $\mathrm{Cd}(50 \mu \mathrm{g} / \mathrm{L})$ induced more obvious inhibition of TCA cycle than the low concentration $(5 \mu \mathrm{g} / \mathrm{L})$ of Cd in clam mitochondria. Overall, the proteomic responses in TCA cycle indicated that mitochondria had to adapt $\mathrm{Cd}$ stimulus by changing its strategy of energy supply.

\subsubsection{Oxidative phosphorylation (OXPHOS)}

Taking place inside mitochondria, oxidative phosphorylation (OXPHOS) provides most of the energy needed for cellular functions. OXPHOS mainly consists of 5 complexes that belong to two sets of reactions, electron transport chain (ETC) and ATP synthesis. In this work, four out of five complexes in OXPHOS, including NADH dehydrogenase [ubiquinone] (complex I), cytochrome bc1 (complex III), cytochrome $c$ oxidative (complex IV) and ATP synthase (complex V), were significantly altered in Cd-treated clam 


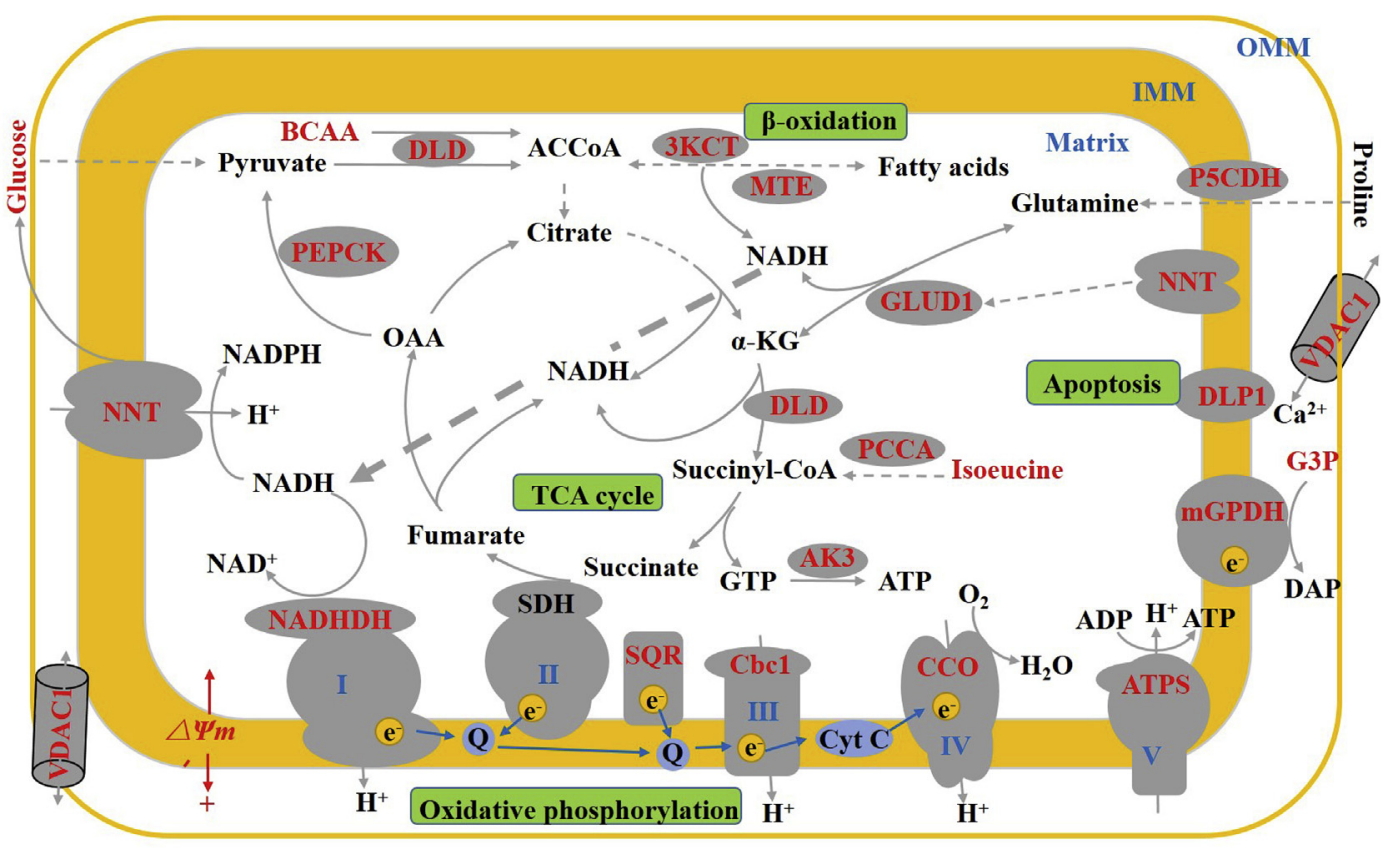

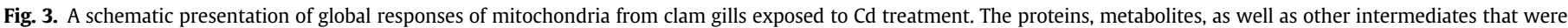

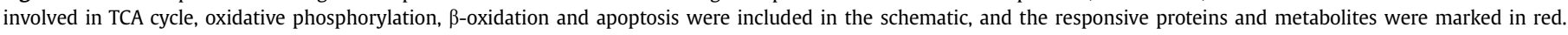

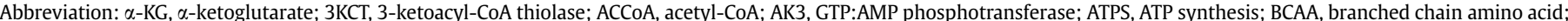

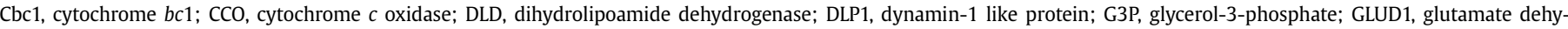

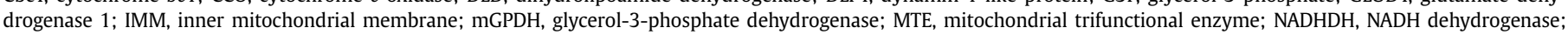

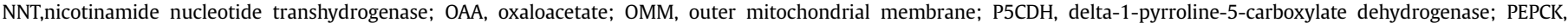
phosphoenolpyruvate carboxykinase; SDH, succinate dehydrogenase; SQR, sulfide quinone oxidoreductase; VDAC1, voltage-dependent anion-selective channel 1.

mitochondria. As the first and largest of complexes of ETC, complex I catalyzes NADH that are generated from TCA cycle and $\beta$-actin into $\mathrm{NAD}^{+}$. In this work, two subunits of complex I were differentially expressed with different tendencies. NADH dehydrogenase [ubiquinone] 1 beta subcomplex subunit 9 is regarded as an accessory subunit of complex I and not involved in catalysis, whereas NADH dehydrogenase [ubiquinone] iron-sulfur protein 3 is one of the core subunits of complex I and belongs to minimal assembly required for catalysis (Murray et al., 2003; Stroud et al., 2016). Complex III was ever regarded as the only site in the ETC complex where ROS were produced in the presence of Cd (Wang et al., 2004). Complex IV catalyzes the transfer of electrons from reduced cytochrome $c$ to molecular oxygen. Complex $\mathrm{V}$ produces ATP from ADP in the presence of a proton gradient (MMP) across the membrane which is generated by electron transport complexes of the respiratory chain. In the low concentration $(5 \mu \mathrm{g} / \mathrm{L})$ of $\mathrm{Cd}$-treated group, six proteins involved in complex I, III, IV and V were altered with inconsistent tendencies, which were even observed in two subunits of one complex. Differing from the low concentration $(5 \mu \mathrm{g} / \mathrm{L})$ of $\mathrm{Cd}-$ treated group, four proteins related to complex I, IV and $\mathrm{V}$ were differentially expressed in the high concentration $(50 \mu \mathrm{g} / \mathrm{L})$ of $\mathrm{Cd}$ treated group. Previous evidences confirmed the toxicological effects of Cd to OXPHOS (Cannino et al., 2009; David et al., 2010; Izrael-Zivkovic et al., 2018; Sokolova et al., 2005; Wang et al., 2004). Especially, Sokolova et al. (2005) observed that Cd exposure significantly decreased ADP-stimulated respiration (state 3), leading to impaired capacity of ATP production. However, some studies have given results differing from what were found in this work. Wang et al. (2004) reported that complex II and III of ETC from pig liver, brain and heart presented greater inhibition by $\mathrm{Cd}$ than the other complex. These inconsistent results of responses to $\mathrm{Cd}$ might suggest the differential mechanisms of $\mathrm{Cd}$ toxicity between marine clams and mammals. Furthermore, some proteins indirectly related to OXPHOS were also found to be differentially expressed in responses to Cd treatments. As a cofactor, NAD is essential in connecting TCA cycle and respiratory chain. Nicotinamide/nicotinic acid mononucleotide adenylyltransferase (NMNAT) is the master enzyme of NAD biosynthesis in living organisms (Zhai et al., 2009). The down-regulation of NMNAT in both Cd treatments indicated that $\mathrm{Cd}$ inhibited the NAD biosynthesis in mitochondria. GTP:AMP phosphotransferase (AK3) contributes to the interconversion of adenine nucleotides and plays an important role in cellular energy homeostasis (Muller et al., 2017). Mitochondrial glycerol-3phosphate dehydrogenase (GPDH) is a flavin-linked respiratory chain dehydrogenase that oxidizes glycerol-3-phosphate (G3P) to dihydroxyacetone phosphate (DAP), and serves as an important enzyme of intermediary metabolism that links glycolysis, oxidative phosphorylation and fatty acid metabolism (Mracek et al., 2013). The down-regulated GPDH in $5 \mu \mathrm{g} / \mathrm{L}$ Cd-treated group was consistent with the elevated G3P that was characterized by metabolomic analysis (Fig. 3, Supporting Information S1, Fig. 1S). Downregulations of these two proteins, including AK3 and GPDH, induced by $5 \mu \mathrm{g} / \mathrm{L} \mathrm{Cd}$ confirmed that energy homeostasis were reconstructed in Cd-treated clams.

\subsubsection{Fatty acid $\beta$-oxidation}

Fatty acid $\beta$-oxidation (FAO) is an important catabolic process by which fatty acids are broken down to generate acetyl-CoA, NADH and $\mathrm{FADH}_{2}$. Then acetyl-CoA enters the TCA cycle, and NADH and $\mathrm{FADH}_{2}$ were used in electron transport chain (ETC) (Wang et al., 2010). Mitochondrial trifunctional enzyme (MTE), also known as hydroxyacyl-CoA dehydrogenase/3-ketoacyl-CoA thiolase/enoylCoA hydratase, is an $\alpha_{2} \beta_{2}$ complex consisting of two types of polypeptides catalyzing three of the four reactions of the $\beta$-oxidation (Venkatesan and Wierenga, 2013). 3-ketoacyl-CoA thiolase (3KCT) functions as an enzyme catalyzing the last step of the 
mitochondrial $\beta$-oxidation pathway (Uchida et al., 1992). In this work, MTE were decreased in both Cd treatment groups, while reduction of $3 \mathrm{KCT}$ were observed in the high concentration $(50 \mu \mathrm{g} /$ L) of Cd-treated group only. The down-regulations of MTE and 3KCT indicated an inhibition of FAO in Cd-treated clams, subsequently leading to decreased supply of acetyl-CoA and NADH to TCA cycle and OXPHOS, respectively.

\subsection{Stress resistance and apoptosis}

As energy powerhouses, mitochondria are also helpful in resistance to stress in organisms especially in intertidal bivalves (Sokolova, 2018). Mitochondrial stress like over-production of ROS not only impedes ATP synthesis via influencing mitochondrial membrane potential and ETC, but also elevates steady-state cytosolic $\mathrm{Ca}^{2+}$ levels (Biswas et al., 1999; Hu and Liu, 2011). Once mitochondria undergo challenges, a serial of responsive proteins will be differentially expressed to safeguard mitochondrial function (Valera-Alberni and Canto, 2018). In addition, the disturbance in mitochondrial dynamics due to long-lasting stress may trigger apoptosis in mitochondria. In this work, alterations of some stressresponsive and apoptosis proteins, including ATP-binding cassette $(A B C)$, aldehyde dehydrogenases 2 (ALDH 2), sulfide quinone oxidoreductase (SQR), ATP-dependent RNA helicase DDX3X (DDX3X), stomatin-like protein 2 (SLP2) and voltage-dependent anion-selective channel 1 (VDAC1) were differentially expressed in Cd-treated clam samples. $\mathrm{ABC}$ belongs to transmembrane proteins and can drive the transport of various molecules across all cell membranes by binding and using ATP. It is usually associated with stress resistance (Carmona-Antonanzas et al., 2015; Chavan et al., 2011). The elevation of $A B C$ implied that $C d$ treatment caused mitochondrial stress in clams. SLP2 functions as regulating the stability of specific mitochondrial proteins, such as respiratory chain complexes (Da Cruz et al., 2008). The up-regulation of SLP2 suggested the instability of ETC complexes induced by $\mathrm{Cd}$, which were confirmed by the alterations of OXPHOS proteins mentioned above. It is noteworthy that up-regulations of ABC and SLP2 were detected in clams from both Cd treatments, indicating that these two proteins might be preferable biomarkers of stress-response to Cd exposure. Other DEPs, such as ALDH2, SQR and DDX3X, were only found to be altered in the low concentration $(5 \mu \mathrm{g} / \mathrm{L})$ of $\mathrm{Cd}-$ treated clam mitochondria. ALDH2 acts as an aldehyde scavenger during lipid peroxidation caused by stress and has ever been found to be elevated in men exposed to high levels of air pollutant like particulate matter (Singh et al., 2013; Winckelmans et al., 2017). The up-regulation of ALDH2 confirmed that there indeed existed stress in clams caused by $\mathrm{Cd}$. SQR is a highly conserved enzyme involved in sulfide metabolism (Brito et al., 2009). It has been reported that SQR played a critical role in Cd detoxification in Schizosaccharomyces pombe (Kennedy et al., 2008). DDX3X is a highly conserved member of ATP-dependent DEAD-box RNA helicases and plays a key role in regulating the mitochondrial stress response (Padmanabhan et al., 2016). In our case, however, neither SQR nor DDX3X showed expected increases in Cd-treated clams, implying the possible dose-dependent effects of Cd to clams.

It is well accepted that apoptosis is closely associated with mitochondria. VDAC1 locates in the mitochondrial outer membrane forms hydrophilic pores and is the main gateway for the entry and exit of the mitochondrial metabolites (Colombini et al., 1996). Apart from being involved in energy metabolism, VDAC1 has an important function in apoptosis (Tomasello et al., 2013). The downregulated VDAC1 in clams revealed the inhibition of apoptosis in $5 \mu \mathrm{g} / \mathrm{L}$ Cd-treated clams. A previous study, however, gave an inconsistent result that higher concentrations $(>3 \mathrm{mg} / \mathrm{L})$ of $\mathrm{Cd}$ significantly induced apoptosis in clams Meretrix meretrix (Xia et al.,
2016).

The unexpected expressions of proteins including SQR, DDX3X, and VDAC1 implied the dose-dependent effects of Cd on stress resistance and apoptosis in clams. Treatments containing more concentrations of $\mathrm{Cd}$ are necessary to further characterize the mechanisms of mitochondrial stress resistance and apoptosis.

\section{Conclusion}

In this work, multiple biological processes in mitochondria, including TCA cycle, oxidative phosphorylation, fatty acid $\beta$ oxidation, stress resistance and apoptosis and mitochondrial fission, were significantly influenced by Cd exposure for 5 weeks. Furthermore, a significant decrease of mitochondrial membrane potential $(\triangle \Psi m)$ and mitochondrial elongations were also observed in Cd-treated clam gills. These findings confirmed that mitochondrion was one of the key targets of Cd toxicity, and the dynamical regulations, such as reconstruction of energy homeostasis, induction of stress resistance and apoptosis, and morphological alterations, in mitochondria might play essential roles in $\mathrm{Cd}$ detoxification. Overall, this work presented a global view of mitochondrial responses of clams to $\mathrm{Cd}$, contributing to better understanding the toxicological mechanisms of $\mathrm{Cd}$.

\section{Acknowledgements}

This research was supported by the grants from National Natural Science Foundation of China (41676114), the Young Taishan Scholars Program of Shandong Province for Prof. Huifeng Wu (tsqn201812115), Qingdao National Laboratory for Marine Science and Technology (QNLM201701) and the Youth Innovation Promotion Association CAS (2015169).

\section{Appendix A. Supplementary data}

Supplementary data related to this article can be found at https://doi.org/10.1016/j.envpol.2019.05.046.

\section{References}

Belyaeva, E.A., Dymkowska, D., Wieckowski, M.R., Wojtczak, L., 2008. Mitochondria as an important target in heavy metal toxicity in rat hepatoma AS-30D cells. Toxicol. Appl. Pharmacol. 231, 34-42.

Biswas, G., Adebanjo, O.A., Freedman, B.D., Anandatheerthavarada, H.K. Vijayasarathy, C., Zaidi, M., Kotlikoff, M., Avadhani, N.G., 1999. Retrograde Ca ${ }^{2+}$ signaling in C2C12 skeletal myocytes in response to mitochondrial genetic and metabolic stress: a novel mode of inter-organelle crosstalk. EMBO J. 18 $522-533$.

Bradford, M.M., 1976. A rapid and sensitive method for the quantitation of microgram quantities of protein utilizing the principle of protein-dye binding. Anal Biochem. 72, 248-254.

Brito, J.A., Sousa, F.L., Stelter, M., Bandeiras, T.M., Vonrhein, C., Teixeira, M., Pereira, M.M., Archer, M., 2009. Structural and functional insights into sulfide: quinone oxidoreductase. Biochemistry 48, 5613-5622.

Calvo, S.E., Mootha, V.K., 2010. The mitochondrial proteome and human disease. Annu. Rev. Genom. Hum. Genet. 11, 25-44.

Cannino, G., Ferruggia, E., Luparello, C., Rinaldi, A.M., 2009. Mitochondrial compartment: a possible target of cadmium effects on breast epithelial cells. Mol. Cell. Biochem. 328, 75-84.

Carmona-Antonanzas, G., Carmichael, S.N., Heumann, J., Taggart, J.B., Gharbi, K. Bron, J.E., Bekaert, M., Sturm, A., 2015. A Survey of the ATP-binding cassette $(\mathrm{ABC})$ gene superfamily in the salmon louse (Lepeophtheirus salmonis). PLoS One 10 e0137394.

Chavan, H., Oruganti, M., Krishnamurthy, P., 2011. The ATP-binding cassette transporter ABCB6 is induced by arsenic and protects against arsenic cytotoxicity. Toxicol. Sci. 120, 519-528.

Chen, S., McKinney, G.J., Nichols, K.M., Colbourne, J.K., Sepulveda, M.S., 2015. Novel cadmium responsive microRNAs in Daphnia pulex. Environ. Sci. Technol. 49, $14605-14613$.

Choong, G., Liu, Y., Templeton, D.M., 2014. Interplay of calcium and cadmium in mediating cadmium toxicity. Chem. Biol. Interact. 211, 54-65.

Colombini, M., Blachly-Dyson, E., Forte, M., 1996. VDAC, a channel in the outer 
mitochondrial membrane. Ion Channels 4, 169-202.

Da Cruz, S., Parone, P.A., Gonzalo, P., Bienvenut, W.V., Tondera, D., Jourdain, A , Quadroni, M., Martinou, J.C., 2008. SLP-2 interacts with prohibitins in the mitochondrial inner membrane and contributes to their stability. BBA-Mol. Cell Res. 1783, 904-911.

David, J.P., Coissac, E., Melodelima, C., Poupardin, R., Riaz, M.A., Chandor-Proust, A. Reynaud, S., 2010. Transcriptome response to pollutants and insecticides in the dengue vector Aedes aegypti using next-generation sequencing technology. BMC Genomics 11, 216-228.

Fernandez-Leborans, G., Herrero, Y.O., 1999. Toxicity and bioaccumulation of cadmium in marine protozoa communities. Ecotoxicol. Environ. Saf. 43, 292-300.

Frank, S., Gaume, B., Bergmann-Leitner, E.S., Leitner, W.W., Robert, E.G., Catez, F. Smith, C.L., Youle, R.J., 2001. The role of dynamin-related protein 1, a mediator of mitochondrial fission, in apoptosis. Dev. Cell 515-525.

Gameiro, P.A., Laviolette, L.A., Kelleher, J.K., Iliopoulos, O., Stephanopoulos, G., 2013 Cofactor balance by nicotinamide nucleotide transhydrogenase (NNT) coordinates reductive carboxylation and glucose catabolism in the tricarboxylic acid cycle (TCA). J. Biol. Chem. 288, 12967-12977.

Gao, X., Zhou, F., Chen, C.T.A., 2014. Pollution status of the Bohai Sea: an overview of the environmental quality assessment related trace metals. Environ. Int. 62 $12-30$.

Gobe, G., Crane, D., 2010. Mitochondria, reactive oxygen species and cadmium toxicity in the kidney. Toxicol. Lett. 198, 49-55.

He, F., DiMario, P.J., 2011. Drosophila delta-1-pyrroline-5-carboxylate dehydrogenase ( $\mathrm{P} 5 \mathrm{CDh}$ ) is required for proline breakdown and mitochondrial integrityEstablishing a fly model for human type II hyperprolinemia. Mitochondrion 11, 397-404.

Hu, F., Liu, F., 2011. Mitochondrial stress: a bridge between mitochondria dysfunction and metabolic diseases? Cell. Signal. 23, 1528-1533.

Izrael-Zivkovic, L., Rikalovic, M., Gojgic-Cvijovic, G., Kazazic, S., Vrvic, M., Brceski, I, Beskoski, V., Loncarevic, B., Gopcevic, K., Karadzic, I., 2018. Cadmium specific proteomic responses of a highly resistant Pseudomonas aeruginosa san ai. RSC Adv. 8, 10549-10560.

Ji, C., Cao, L., Li, F., 2015b. Toxicological evaluation of two pedigrees of clam Ruditapes philippinarum as bioindicators of heavy metal contaminants using metabolomics. Environ. Toxicol. Pharmacol. 39, 545-554.

Ji, C., Wu, H., Zhou, M., Zhao, J., 2015a. Multiple biomarkers of biological effects induced by cadmium in clam Ruditapes philippinarum. Fish Shellfish Immunol. 44, 430-435.

Ji, C., Yu, D., Wang, Q., Li, F., Zhao, J., Wu, H., 2016. Impact of metal pollution on shrimp Crangon affinis by NMR-based metabolomics. Mar. Pollut. Bull. 106, $372-376$

Kanamaru, Y., Sekine, S., Ichijo, H., Takeda, K., 2012. The phosphorylationdependent regulation of mitochondrial proteins in stress responses. J. Signa Transduction 2012, 931215.

Kennedy, P.J., Vashisht, A.A., Hoe, K.L., Kim, D.U., Park, H.O., Hayles, J., Russell, P., 2008. A genome-wide screen of genes involved in cadmium tolerance in Schizosaccharomyces pombe. Toxicol. Sci. 106, 124-139.

Li, J., Cai, T., Wu, P., Cui, Z., Chen, X., Hou, J., Xie, Z., Xue, P., Shi, L., Liu, P., Yates, J.R., Yang, F., 2009. Proteomic analysis of mitochondria from Caenorhabditis elegans. Proteomics 9, 4539-4553.

Li, J., Huang, Q., Long, X., Guo, X., Sun, X., Jin, X., Li, Z., Ren, T., Yuan, P., Huang, X. Zhang, H., Xing, J., 2017. Mitochondrial elongation-mediated glucose metabolism reprogramming is essential for tumour cell survival during energy stress, Oncogene 36, 4901-4912.

Li, M., Xia, T., Jiang, C., Li, L., Fu, J., Zhou, Z., 2003. Cadmium directly induced the opening of membrane permeability pore of mitochondria which possibly involved in cadmium-triggered apoptosis. Toxicology 194, 19-33.

Liu, X., Zhang, L., You, L., Yu, J., Cong, M., Wang, Q., Li, F., Li, L., Zhao, J., Li, C., Wu, H., 2011. Assessment of clam Ruditapes philippinarum as heavy metal bioindicators using NMR-based metabolomics. Clean. - Soil, Air, Water 39, 759-766.

Luo, W., Lu, Y., Wang, T. Kong, P.R., Jiao, W., Hu, W., Jia, J., Naile, J.E., Khim, J.S., Giesy, J.P., 2013. Environmental concentrations and bioaccumulations of cadmium and zinc in coastal watersheds along the Chinese Northern Bohai and Yellow Seas. Environ. Toxicol. Chem. 32, 831-840.

Ma, J., Shao, M., Bao, Y., Yin, J., 1995. Current status and assessment of bays of the Bohai Sea and huanghai sea in liaoning Province. Res. Environ. Sci. 8, 27-34 ([In Chinese with English abstract]).

Mai, S., Klinkenberg, M., Auburger, G., Bereiter-Hahn, J. Jendrach, M., 2010 Decreased expression of Drp1 and Fis1 mediates mitochondrial elongation in senescent cells and enhances resistance to oxidative stress through PINK1. J. Cell Sci. 123, 917-926.

Marques, A., Piló, D., Carvalho, S., Araújo, O., Guilherme, S., Santos, M.A., Vale, C. Pereira, F., Pacheco, M., Pereira, P., 2018. Metal bioaccumulation and oxidative stress profiles in Ruditapes philippinarum - insights towards its suitability as bioindicator of estuarine metal contamination. Ecol. Indicat. 95, 1087-1099.

Meyer, J.N., Leung, M.C.K., Rooney, J.P., Sendoel, A., Hengartner, M.O., Kisby, G.E., Bess, A.S., 2013. Mitochondria as a target of environmental toxicants. Toxicol Sci. 134, 1-17.

Mracek, T., Drahota, Z., Houstek, J., 2013. The function and the role of the mitochondrial glycerol-3-phosphate dehydrogenase in mammalian tissues. BBABioenerg. 1827, 401-410.

Muller, L., 1986. Consequences of cadmium toxicity in rat hepatocytes: mitochondrial dysfunction and lipid peroxidation. Toxicology 40, 285-295.

Muller, W.E.G., Wang, S.F., Neufurth, M., Kokkinopoulou, M., Feng, Q.L.
Schroder, H.C., Wang, X.H., 2017. Polyphosphate as a donor of high-energy phosphate for the synthesis of ADP and ATP. J. Cell Sci. 130, 2747-2756.

Murray, J., Zhang, B., Taylor, S.W., Oglesbee, D., Fahy, E., Marusich, M.F., Ghosh, S.S., Capaldi, R.A., 2003. The subunit composition of the human NADH dehydrogenase obtained by rapid one-step immunopurification. J. Biol. Chem. 278, $13619-13622$.

Padmanabhan, P.K., Zghidi-Abouzid, O., Samant, M., Dumas, C., Aguiar, B.G., Estaquier, J., Papadopoulou, B., 2016. DDX3 DEAD-box RNA helicase plays a central role in mitochondrial protein quality control in Leishmania. Cell Death Dis. 7 e2406.

Padmini, E., Rani, M.U., 2011. Mitochondrial membrane potential is a suitable candidate for assessing pollution toxicity in fish. Sci. Total Environ. 409, 3687-3700.

Qu, R.J., Wang, X., Feng, M., Li, Y., Liu, H., Wang, L., Wang, Z., 2013. The toxicity of cadmium to three aquatic organisms (Photobacterium phosphoreum, Daphnia magna and Carassius auratus) under different pH levels. Ecotoxicol. Environ. Saf. 95, 83-90.

Rasheed, B.K.A., Diwan, J.J., Sanadi, D.R., 1984. Activation of potassium-ion transport in mitochondria by cadmium ion. Eur. J. Biochem. 144, 643-647.

Singh, S., Brocker, C., Koppaka, V., Chen, Y., Jackson, B.C., Matsumoto, A., Thompson, D.C., Vasiliou, V., 2013. Aldehyde dehydrogenases in cellular responses to oxidative/electrophilic stress. Free Radical Biol. Med. 56, 89-101.

Sokolova, I., 2018. Mitochondrial adaptations to variable environments and their role in animals' stress tolerance. Integr. Comp. Biol. 58, 519-531.

Sokolova, I.M., 2004. Cadmium effects on mitochondrial function are enhanced by elevated temperatures in a marine poikilotherm, Crassostrea virginica Gmelin (Bivalvia : ostreidae). J. Exp. Biol. 207, 2639-2648.

Sokolova, I.M., Sokolov, E.P., Ponnappa, K.M., 2005. Cadmium exposure affects mitochondrial bioenergetics and gene expression of key mitochondrial proteins in the eastern oyster Crassostrea virginica Gmelin (Bivalvia: ostreidae). Aquat. Toxicol. 73, 242-255.

Stark, R., Guebre-Egziabher, F., Zhao, X., Feriod, C., Dong, J.Y., Alves, T.C., Ioja, S., Pongratz, R.L., Bhanot, S., Roden, M., Cline, G.W., Shulman, G.I., Kibbey, R.G., 2014. A role for mitochondrial phosphoenolpyruvate carboxykinase (pepck-m) in the regulation of hepatic gluconeogenesis. J. Biol. Chem. 289, 7257-7263.

Stroud, D.A., Surgenor, E.E., Formosa, L.E., Reljic, B., Frazier, A.E., Dibley, M.G., Osellame, L.D., Stait, T., Beilharz, T.H., Thorburn, D.R., Salim, A., Ryan, M.T. 2016 Accessory subunits are integral for assembly and function of human mitochondrial complex I. Nature 538, 123-126.

Timmins-Schiffman, E., Coffey, W.D., Hua, W., Nunn, B.L., Dickinson, G.H., Roberts, S.B., 2014. Shotgun proteomics reveals physiological response to ocean acidification in Crassostrea gigas. BMC Genomics 15, 591.

Tomasello, M.F., Guarino, F., Reina, S., Messina, A., De Pinto, V., 2013. The voltagedependent anion selective channel 1 (VDAC1) topography in the mitochondrial outer membrane as detected in intact cell. PLoS One 8 e81522.

Uchida, Y., Izai, K., Orii, T., Hashimoto, T., 1992. Novel fatty acid beta-oxidation enzymes in rat liver mitochondria. II. Purification and properties of enoylcoenzyme A (CoA) hydratase/3-hydroxyacyl-CoA dehydrogenase/3-ketoacylCoA thiolase trifunctional protein. J. Biol. Chem. 267, 1034-1041.

Valera-Alberni, M., Canto, C., 2018. Mitochondrial stress management: a dynamic journey. Cell Stress 2, 253-274.

Venkatesan, R., Wierenga, R.K., 2013. Structure of mycobacterial beta-oxidation trifunctional enzyme reveals its altered assembly and putative substrate channeling pathway. ACS Chem. Biol. 8, 1063-1073.

Vincent, E.E., Sergushichev, A., Griss, T., Gingras, M.C., Samborska, B., Ntimbane, T., Coelho, P.P., Blagih, J., Raissi, T.C., Choiniere, L., Bridon, G., Loginicheva, E., Flynn, B.R., Thomas, E.C., Tavare, J.M., Avizonis, D., Pause, A., Elder, D.J.E., Artyomov, M.N., Jones, R.G., 2015. Mitochondrial phosphoenolpyruvate carboxykinase regulates metabolic adaptation and enables glucose-independent tumor growth. Mol. Cell 60, 195-207.

Wang, W.-X., Rainbow, P.S., 2006. Subcellular partitioning and the prediction of cadmium toxicity to aquatic organisms. Environ. Chem. 3, 395-399.

Wang, Y., Fang, J., Leonard, S.S., Rao, K.M.K., 2004. Cadmium inhibits the electron transfer chain and induces reactive oxygen species. Free Radical Biol. Med. 36, $1434-1443$

Wang, Y., Mohsen, A.W., Mihalik, S.J., Goetzman, E.S., Vockley, J., 2010. Evidence for physical association of mitochondrial fatty acid oxidation and oxidative phosphorylation complexes. J. Biol. Chem. 285, 29834-29841.

Winckelmans, E., Nawrot, T.S., Tsamou, M., Den Hond, E., Baeyens, W., Kleinjans, J., Lefebvre, W., Van Larebeke, N., Peusens, M., Plusquin, M., Reynders, H., Schoeters, G., Vanpoucke, C., de Kok, T.M., Vrijens, K., 2017. Transcriptome-wide analyses indicate mitochondrial responses to particulate air pollution exposure. Environ. Health 16, 87.

Wongkittichote, P., Mew, N.A., Chapman, K.A., 2017. Propionyl-CoA carboxylase-A review. Mol. Genet. Metab. 122, 145-152.

Wu, H., Xu, L., Yu, D., Ji, C., 2017. Differential metabolic responses in three life stages of mussels Mytilus galloprovincialis exposed to cadmium. Ecotoxicology 26, $74-80$.

Xia, L., Chen, S., Dahms, H.-U., Ying, X., Peng, X., 2016. Cadmium induced oxidative damage and apoptosis in the hepatopancreas of Meretrix meretrix. Ecotoxicology 25, 959-969.

Xu, L., Ji, C., Zhao, J., Wu, H., 2016a. Metabolic responses to metal pollution in shrimp Crangon affinis from the sites along the Laizhou Bay in the Bohai Sea. Mar. Pollut. Bull. 113, 536-541.

Xu, L., Peng, X., Yu, D., Ji, C., Zhao, J., Wu, H., 2016b. Proteomic responses reveal the 
differential effects induced by cadmium in mussels Mytilus galloprovinvialis at early life stages. Fish Shellfish Immunol. 55, 510-515.

Xu, S., Pi, H., Chen, Y., Zhang, N., Guo, P., Lu, Y., He, M., Xie, J., Zhong, M., Zhang, Y. Yu, Z., Zhou, Z., 2013. Cadmium induced Drp1-dependent mitochondrial fragmentation by disturbing calcium homeostasis in its hepatotoxicity. Cell Death Dis. 4 e540.

Yang, C., Ko, B., Hensley, C.T., Jiang, L., Wasti, A.T., Kim, J., Sudderth, J., Calvaruso, M.A., Lumata, L., Mitsche, M., Rutter, J., Merritt, M.E. DeBerardinis, R.J., 2014. Glutamine oxidation maintains the TCA cycle and cell survival during impaired mitochondrial pyruvate transport. Mol. Cell 56, 414-424.

Yoon, Y., Pitts, K.R., Dahan, S., McNiven, M.A., 1998. A novel dynamin-like protein associates with cytoplasmic vesicles and tubules of the endoplasmic reticulum in mammalian cells. J. Cell Biol. 140, 779-793.

Youle, R.J., van der Bliek, A.M., 2012. Mitochondrial fission, fusion, and stress.
Science 337, 1062-1065.

Yu, T., Deuster, P., Chen, Y., 2016. Role of dynamin-related protein 1-mediated mitochondrial fission in resistance of mouse C2C12 myoblasts to heat injury. J. Physiol.-London 594, 7419-7433.

Zhai, R.G., Rizzi, M., Garavaglia, S., 2009. Nicotinamide/nicotinic acid mononucleotide adenylyltransferase, new insights into an ancient enzyme. Cell. Mol. Life Sci. 66, 2805-2818.

Zhang, X., 2001. Investigation of pollution of $\mathrm{Pb}, \mathrm{Cd}, \mathrm{Hg}$, as in sea water and deposit of the Bohai Sea area. Heilongjiang Environ. J. 25, 87-90 ([In Chinese with English abstract])

Zorova, L.D., Popkov, V.A., Plotnikov, E.Y., Silachev, D.N., Pevzner, I.B., Jankauskas, S.S., Babenko, V.A., Zorov, S.D., Balakireva, A.V., Juhaszova, M. Sollott, S.J., Zorov, D.B., 2018. Mitochondrial membrane potential. Anal. Biochem. 552, 50-59. 